\title{
Palliative care and quality of life in neuro-oncology
}

\section{Naveen Mummudi and Rakesh Jalali*}

\author{
Address: Department of Radiation Oncology, 1129, Homi Bhabha Block, Tata Memorial Hospital, Parel, Mumbai - 400012, India \\ *Corresponding author: Rakesh Jalali (rjalali@tmc.gov.in) \\ Fl000Prime Reports 2014, 6:7I (doi:10.12703/P6-7I) \\ All Fl000Prime Reports articles are distributed under the terms of the Creative Commons Attribution-Non Commercial License \\ (http://creativecommons.org/licenses/by-nc/3.0/legalcode), which permits non-commercial use, distribution, and reproduction in any medium, \\ provided the original work is properly cited. \\ The electronic version of this article is the complete one and can be found at: http://f $1000 . c o m / p r i m e /$ reports $/ \mathrm{m} / 6 / 7$ I
}

\begin{abstract}
Health-related quality of life has become an important end point in modern day clinical practice in patients with primary or secondary brain tumors. Patients have unique symptoms and problems from diagnosis till death, which require interventions that are multidisciplinary in nature. Here, we review and summarize the various key issues in palliative care, quality of life and end of life in patients with brain tumors, with the focus on primary gliomas.
\end{abstract}

\section{Introduction}

"Palliative care begins from the understanding that every patient has his or her own story, relationships and culture, and is worthy of respect as a unique individual. This respect includes giving the best available medical care and making the advances of recent decades fully available." - Dame Cecily Saunders.

Primary and secondary malignant tumors of the brain, despite combined modality treatment with surgery, radiotherapy, and chemotherapy, are virtually incurable; palliation, maintenance and improvement of the patient's quality of life is of more importance. From diagnosis to the end of life, the care needs of patients with brain tumors are high, underestimated and often neglected. Also, care and needs increase towards the end of life, with a high incidence of neurological symptoms and psychosocial problems [1].

Palliative care, as defined by the World Health Organization, is "an approach that improves the quality of life of patients and their families facing the problems associated with life-threatening illness, through the prevention and relief of suffering by means of early identification and impeccable assessment and treatment of pain and other problems, physical, psychosocial and spiritual". The goal of palliative care is to maximize quality of life, with inputs from a multidisciplinary team to help the patient live as actively as possible whilst neither hastening nor postponing death [2]. Early integration of palliative care into the treatment schedule improves quality of life and symptom management, and leads to a reduction in aggressive therapy at the end of life $[3,4]$.

Patients diagnosed with brain tumors and their caregivers undergo enormous physical, emotional, social and financial hardships, during diagnosis, treatment and towards the end of life.

\section{Quality of life issues}

Quality of life is a concept that encompasses the multidimensional well-being of a person in terms of physical or functional status, as well as emotional and social wellbeing, and therefore reflects an individual's overall satisfaction with life [5]. Health-related quality of life is, by definition, a patient-reported outcome measure, reflecting the patient's perspective.

Studies on quality of life are primarily qualitative and have focused on specific symptoms such as fatigue, sleep disorders, cognitive dysfunction and some symptom clusters. Quality of life in brain tumor patients is complex and multidimensional in nature, with symptoms having interrelationships with each other as well as with patient, tumor, and treatment factors. The increased interest in exploring quality of life as a primary end point for cancer therapy has created a need for prospective, controlled studies to assess baseline and serial quality of life 
parameters apart from the classic outcome measures, such as progression-free survival and overall survival [5]. In fact, in the RTOG-0825 study [6], the baseline and early change in neurocognitive function were prognostic for overall survival and progression-free survival. However, assessing quality of life is challenging as validated instruments for measurements are scarce, serial measurements over time are often associated with a lack of compliance, and there is a lack of well-designed trials.

\section{Quality of life measurement}

Impairments are the direct consequences of disease, demonstrated by physical examination, and can be evaluated using neurological and neuropsychological examinations. Disability is the impact of this impairment on the patient's ability to carry out activities and can be determined using scales such as the Barthel index, and the Karnofsky Performance Status Scale. Handicap is the consequence of disability on patient well-being; the Modified Rankin Handicap Scale and the Spitzer scale are specific handicap scales for brain tumor patients [7].

Various health-related quality of life measures are available for use in clinical trials as well as in daily clinical work. The European Organization for Research and Treatment of Cancer quality of life questionnaire (EORTC QLQ-C30) consists of 30 items, which are organized into five functional scales (physical, role, emotional, cognitive and social functioning), three symptom scales (fatigue, nausea and vomiting, and pain), one global health status scale, one overall quality of life scale, and six single items (dyspnoea, insomnia, appetite loss, constipation, diarrhoea, and financial difficulties) [8]. This core questionnaire can be supplemented with a brain tumor-specific questionnaire, the EORTC QLQ-BN20, which includes 20 items, which are organized into four scales (future uncertainty, visual disorders, motor dysfunction, and communication deficit) and seven single items (headache, seizures, drowsiness, hair loss, itchy skin, weakness of legs, and bladder control) [9]. All single item and/or multi-item scales of the EORTC questionnaires are linearly transformed to $0-100$ scales. Change in scores of $\geq 10$ points on any given scale are interpreted as being clinically meaningful; changes of $>20$ points represent a very large effect. The Functional Assessment of Cancer Therapy-General questionnaire (FACT-G) consists of 27 items covering four domains: physical, social/family, emotional, and functional wellbeing [10]. A brain cancer-specific subscale consists of 23 items measuring concerns relevant to patients with brain tumors [11]. The EORTC measures are more focused on functioning and symptoms, while the FACT measures cover more psychosocial aspects of the disease and its treatment. The recent M.D. Anderson Symptom Inventory (MDASI) questionnaire was specifically designed to measure the severity of symptoms in cancer patients (13 items) as well as the interference of these symptoms with activities of daily living (six items) [12]. In addition to the core questionnaire, a brain tumor-specific module (MDASI-BT) has been developed, consisting of nine items (weakness, difficulty in understanding and speaking, seizures, difficulty concentrating, vision, change in appearance, change in bowel pattern, and irritability) [13].

Proxies or health care professionals can rate patient quality of life, when patients are unable to self-report. Proxies and health care providers tend to report more health-related quality of life problems than do patients themselves and proxy ratings tend to be more in agreement with patient physical health-related quality of life domains than with psychological domains [7].

\section{Specific symptoms}

Brain tumor patients deal with a significant symptom burden. Studies that have evaluated specific symptoms affecting quality of life have been mostly descriptive, and most examine a heterogeneous group of patients receiving different therapies at different stages of their illness. Most of these studies have focused on fatigue, sleep, pain, seizures, mood disturbance and cognitive function [14-16]. While some of these symptoms are seen in patients with other malignancies, fatigue, neurological deficits, seizure, cognitive dysfunction and mood disturbances are specifically encountered in neuro-oncology.

Fatigue is one of the significant symptoms in patients with newly diagnosed and recurrent high-grade gliomas and may be more significant a problem when compared to patients with low-grade tumors [17-19]. While seizures are more commonly observed in low-grade glioma patients than in high-grade glioma patients, and are associated with deterioration in multiple cognitive domains, the prognostic impact of seizures remains contentious [20-22].

Mood disturbances, especially anxiety and depression, are commonly noted in brain tumor patients [5]. Depression is one of the most important independent predictors of quality of life and has been shown to have an adverse impact on survival $[23,24]$. Cognitive functioning has also been extensively studied and reported in brain tumor patients [25-27]. Impaired cognition is seen in patients prior to therapy, after radiotherapy and chemotherapy, as well as in patients with tumor recurrence. In a study, cognitive deterioration was detected 6 weeks prior to radiographic failure [28].

Although symptoms such as anxiety, depression, pain, fatigue, and sleep disturbance have been studied 
separately, they are often interrelated and may have a common etiology. Research concerning such symptom clusters in primary brain tumor patients is, however, limited. Pharmacological, non-pharmacological and complementary medicine interventions are being studied to assess their ability to improve cognition and mood disturbances, and hence improve the quality of life [29-32].

\section{Factors influencing quality of life}

Quality of life scores depend on various patient, tumor, and treatment-related factors [33]. There may be differential importance of various factors influencing quality of life scores in high-grade gliomas and low-grade gliomas.

\section{Patient factors}

Performance status is related to quality of life in patients with newly diagnosed high-grade gliomas [34], with a worse Eastern Cooperative Oncology Group (ECOG) performance score associated with worse overall quality of life. In a study evaluating the factors influencing the activities of daily life, the functional independence and functional activity measurement systems were found to be significantly higher in patients with a Karnofsky performance score of 70 or more and a neurological performance scale of 0 or 1 [35]. Some studies of patients with brain tumors reported higher levels of mood disturbance and lower quality of life scores in women than in men [36].

\section{Tumor factors}

Several tumor-related factors like tumor grade, location and laterality have been shown to have an impact on health-related quality of life. Patients with high-grade gliomas experience worse quality of life than patients who have low-grade gliomas $[7,37]$. Depression may also be more frequent in patients with left-hemisphere tumor high-grade gliomas, while right-hemisphere primary brain tumor patients may have higher anxiety. Low-grade glioma patients with lesions in the frontal lobe or lesions in the temporo-parietal lobe are known to have mood disturbances. Also, greater cognitive disability has been noted in those with tumors in the dominant hemisphere $[18,38]$.

\section{Treatment factors}

Treatment modalities like surgery, radiation, chemotherapy and concomitant medications can affect the overall quality of life of brain tumor patients. In high-grade glioma patients, those only undergoing biopsy have worse quality of life than those who have undergone gross total resection [39]. Both radiation-induced fatigue and cognitive dysfunction are known to adversely affect quality of life. Also, cognitive functioning is significantly more impaired in patients receiving whole brain irradiation compared to partial brain irradiation [40]. Patients receiving temozolomide develop more symptoms such as vomiting, anorexia, constipation, and decreased social functioning; the quality of life in newly diagnosed glioblastoma patients receiving either radiotherapy alone or radiotherapy with concomitant and adjuvant temozolomide was substantially impaired compared to historical controls, but no significant decrease in overall quality of life was noted throughout treatment [15].

Bevacizumab, an anti-vascular endothelial growth factor (VEGF) inhibitor, has been studied extensively in recurrent and newly diagnosed glioblastoma patients. In the AVAglio study [41], in addition to significantly prolonging progression-free survival and decreased corticosteroid dependence, bevacizumab was also found to improve or stabilize health-related quality of life compared with placebo; while in the RTOG-0825 study [6], the visuomotor measure of executive function was worse in the bevacizumab arm than the placebo. The contrasting findings related to quality of life between these two large randomized trials could be due to the different parameters evaluated, statistics involved in analysis, and the level of participation in the testing.

Antiepileptic drugs and steroid medications are commonly prescribed to brain tumor patients and can adversely affect physical, emotional, and cognitive functioning. Antiepileptics have been associated with cognitive dysfunction and steroids have been linked to depression in high-grade glioma patients $[20,42]$.

\section{Quality of death and end-of-life issues}

When the patient's condition declines due to tumor progression, and further tumor-directed treatment is not an option, the end-of-life phase begins. During this phase, symptom burden becomes high and patients are often troubled by seizures and deficits in cognition, communication, and motor function. Furthermore, loss of consciousness, cognitive disturbances, communication deficits, and confusion often hamper the patient's competence to participate in end-of-life decision making [43,44].

The goal of end-of-life care is to achieve a death with dignity. The intrinsic dignity of every human being is based on their sense of worth, personal goals and social circumstances; personal dignity can be influenced by various factors such as symptom distress, acceptance of disease, level of independence, spiritual well-being, preservation of social role and social support [45]. A recent study found that "death with dignity" was associated with better communication, especially regarding prognosis, 
and patients being prepared for death. It was also associated with greater satisfaction with the physician providing end-of-life care on the part of relatives [43].

In order to allow the patient to experience a peaceful death, specific palliative interventions are required for the control of pain, confusion, agitation, delirium or seizures. The lack of control of symptoms can often lead to re-hospitalization with a resultant increase in costs and a worsening of patient's quality of life. In a study of over 5000 patients with glioblastoma, more than one fifth were found to be hospitalized for at least $25 \%$ of their remaining lives [46]. Although, hospitalizations are primarily aimed at identifying correctable causes and reducing the symptoms, it may also be emotionally and financially counter-productive; this is true especially in advanced cancer, where hospitalization has been shown to be associated with a reduced quality of life [47]. Many patients prefer to die at home and, in a study on high-grade glioma patients, less than $5 \%$ of patients were actually in favour of hospitalization near the end of life [48]. With effective home-based palliative care, the rate and cost of hospitalization can be significantly lowered [49].

\section{Management of symptoms}

The main goals of palliative care and end-of-life care in patients with brain tumors are to offer adequate symptom control, relief of suffering, to avoid inappropriate prolongation of dying and to support the psychological and spiritual needs of patients and families [50,51].

Epilepsy appears to be one of the more frequent symptoms in the last stage of disease and represents a major issue in the management of dying patients, particularly in those assisted at home. Loss of seizure control in the end-of-life phase may influence the quality of life of patients and their caregivers. In a recent study on high-grade gliomas, more than $35 \%$ of patients developed seizures in the last month before death, with the risk higher in patients with a previous history of epilepsy [52]. Most patients may encounter swallowing difficulties when taking anticonvulsants orally, due to dysphagia and disorientation, hence anticonvulsant therapy needs to be optimized; alternate routes of drug administration (such as intramuscular, rectal, trans-dermal, or subcutaneous) can be considered.

Agitation and restlessness together with physical pain are common features and require appropriate treatment. In the large majority of patients, headache is due to increased intracranial pressure, and usually responds to steroid treatment. In patients with meningeal syndrome due to meningeal involvement, headaches may be severe; steroids, pain medication with non-opioids or opioids might be indicated [53].
In the last weeks of life, most patients experience a progressive loss of consciousness, lethargy, and confusion, and the majority of patients enter into deep coma in the last days. Agitation, delirium and confusion without a complete loss of consciousness may be very distressing for patients and their families, especially in a home care setting. Palliative sedation is the intentional lowering of the level of consciousness of a patient in the last phase of life by administration of sedatives. The objective is to relieve severe physical or psychological suffering that is otherwise untreatable. A subcutaneous infusion of midazolam is used for continuous sedation, if feasible; otherwise intermittent administration of midazolam, diazepam, lorazepam or chlorpromazine may be considered [54].

In the unconscious dying patient, difficulty in clearing upper airways leads to an accumulation of respiratory tract secretions. This "death rattle" may be very distressing for the family and caregivers but is unlikely to be distressing for the patient, owing to the decreased level of consciousness. Gentle nasal suction, postural drainage and administration of anti-cholinergic drugs can help in reducing these symptoms.

\section{End-of-life treatment decisions}

End-of-life treatment decisions in neuro-oncology include withdrawing or withholding of medications, nutritional support and palliative sedation. While withholding medication is a planned decision not to undertake symptomatic therapy that is otherwise warranted, withdrawal is the discontinuation of symptomatic treatments, and terminal sedation is defined as the pharmacologically induced reduction of vigilance up to the point of the complete loss of consciousness, with the aim of reducing or abolishing the perception of symptoms that would otherwise be intolerable $[53,55]$. The process of end-of-life decision making is complicated by the presence of cognitive deterioration that may affect patients' competence to express treatment preferences. Therefore, it is of paramount importance to plan and discuss end-of-life decisions in advance with the patient and the family.

\section{Diversity amongst countries, professionals and cultures}

In many parts of the world, hospice and palliative care is still non-existent or in its infancy. Approximately one million individuals die each week around the globe and, even in developed countries, medical services have often focused on preventing death rather than helping people suffering from pain, discomfort and stress [56].

The International Observatory on End of Life Care (IOELC) reviewed and compared the hospice-palliative 
care activity in various countries. The countries were placed in six different groups based on their activity: no known hospice-palliative care activity, capacity building activity, isolated and generalized hospice-palliative care provision, countries where hospice-palliative care services are at a stage of preliminary integration into mainstream service provision, and countries at a stage of advanced integration into mainstream service provision. In 2006, 115 of the world's 234 countries (49\%) had established one or more hospice-palliative care services. The trend increased by $9 \%$ in 2011: 136 of the world's 234 countries (58\%) had one or more hospice-palliative care service established. In 2006, 156 countries (67\%) were actively engaged in delivering a hospice-palliative care service or developing the framework; by 2011 there had been a slight increase in this number to 159 countries. Also, in most regions of the world, a strong association exists between palliative care and human development, with the more developed countries in the process of integration of palliative care services [57,58].

This heterogeneity across the various countries is highlighted in the study by the Economist Intelligence Unit's research team, which devised the Quality of Death Index by collating data across 40 countries from interviewing various health professionals. The index used differential weightage for basic healthcare environment, availability, quality and cost of end of life care perceived amongst these countries. The bottom-ranked countries in the Quality of Death Index included developing countries, such as China, Mexico, Brazil, India and Uganda, and, surprisingly, some developed countries like Denmark, Japan, Italy, Finland and South Korea. The report also noted that the public awareness regarding end-of-life care was found to be lacking in both developed and developing countries alike [59].

Strong taboos against talking about death exist in various cultures and communities. Even in India, where death is discussed more openly as the inevitable consequence of life, the protective attitude of the relatives still presents a big barrier to open communication with the patient. In the case of children, these taboos around death and dying are stronger. Death of children is more accepted in developing countries with high infant mortality rates, but in the developed world, the "cure at all cost" attitude of the health care professionals and parents heavily influences the endof-life care. This ideological difference owing to specific cultures was evident when we conducted a survey amongst various neuro-oncology professionals in Asian countries; we noticed that most health care professionals refer patients only when they develop symptoms that require palliation, and the referral of patients to hospice care at the end of life was done rarely [60].
A government-led national palliative care strategy exists in very few developed countries. While government policy statements do not necessarily guarantee quality and availability of end-of-life care, they can be valuable if backed up by the development of strategic services. According to the World Health Organisation, about five billion people have insufficient or no access to medications to control severe or moderate pain. While legally any physician can prescribe opioids for pain control, their availability is a major concern in many countries, essentially because of the complex narcotics laws restricting the sale of morphine, as governments are concerned about illicit drug use [59].

\section{Caregivers' perspective}

The diagnosis of a brain tumor has a catastrophic effect not only on the patient but also on the family members. Family caregivers provide extraordinary uncompensated care involving significant amounts of time and energy for months or years, requiring the performance of tasks that are often physically, emotionally, socially, or financially demanding. They are constantly challenged to solve problems and make decisions as care needs change; because the focus is on the patient, their own needs are often neglected [61].

For parents, the grieving process starts right at the diagnosis of the brain tumor and the decision to move toward palliative care is a difficult one, filled with many highly charged emotions including anger, and a search for answers, the intensity of which differs between family members. The neurologic deterioration that characterizes the dying trajectory of children warrants the need for increased awareness of the distinct issues in the palliative care of children with brain tumors and for early anticipatory guidance provided for families. In one study [62], parents described the loss of the ability to communicate as a turning point that led to acceptance. Parental coping mechanisms included striving to maintain normality, and finding spiritual strength through hope and the resilience of their child. Parents are also required to handle routine tasks while learning new skills, involving hands-on patient care, and their stress is compounded by financial hardships, and inadequate community support. Parents of dying children have an overwhelming feeling of loss during the end-of-life phase, and part of the purpose of palliative care is to help them come to terms with the loss of their child. This process is a gradual relinquishing of the instinct to preserve the child's life regardless of their condition and accepting inevitable loss. A perceived loss of control by the parents makes this process a major challenge. However, parents who make this transition are more receptive to their child's needs [63]. 


\section{Conclusion}

In summary, with growing awareness amongst clinicians regarding the quality of life, even in patients with as difficult a disease as brain tumor, focus is shifting towards meaningful prolongation of life. This in turn necessitates an active participation of the patient, family and caregivers, with the health care professional involved in every step of the disease management. "Quality of death" is a concept that could be viewed as a natural extension of quality of life. With the amalgamation of these concepts into routine clinical practice, the basic "right to a decent life" is fortified.

\section{Abbreviations}

EORTC, European Organization for Research and Treatment of Cancer; FACT, Functional Assessment of Cancer Therapy; MDASI, M.D. Anderson Symptom Inventory.

\section{Disclosures}

The authors declare that they have no disclosures.

\section{References}

I. Dirven L, Aaronson NK, Heimans JJ, Taphoorn, Martin JB: Healthrelated quality of life in high-grade glioma patients. Chin J Cancer 2014, 33:40-5.

2. Lin E, Rosenthal MA, Le BH, Eastman P: Neuro-oncology and palliative care: a challenging interface. Neuro-oncology 2012, 14(Suppl 4):iv3-7.

3. Temel JS, Greer JA, Muzikansky A, Gallagher ER, Admane S, Jackson VA, Dahlin CM, Blinderman CD, Jacobsen J, Pirl WF, Billings JA, Lynch TJ: Early palliative care for patients with metastatic non-small-cell lung cancer. N Engl J Med 20I0, 363:733-42.

\section{FlOOOPrime}

\section{RECOMMENDED}

4. Greer JA, Jackson VA, Meier DE, Temel JS: Early integration of palliative care services with standard oncology care for patients with advanced cancer. CA Cancer J Clin 2013, 63:349-63.

\section{FlOOOPrime}

\section{RECOMMENDED}

5. Liu R, Page M, Solheim K, Fox S, Chang SM: Quality of life in adults with brain tumors: current knowledge and future directions. Neuro-oncology 2009, I I:330-9.

6. Wefel JS, Pugh SL, Armstrong TS, Gilbert MR, Won M, Wendland MM, Brachman D, Komaki R, Crocker IR, Robins HI, Lee RJ, Mehta MP: Neurocognitive function (NCF) outcomes in patients with glioblastoma (GBM) enrolled in RTOG 0825. J Clin Oncol 2013, 3 I (Suppl I5):2004.

7. Taphoorn, Martin JB, Sizoo EM, Bottomley A: Review on quality of life issues in patients with primary brain tumors. Oncologist 2010, 15:618-26.

8. Aaronson NK, Ahmedzai S, Bergman B, Bullinger M, Cull A, Duez NJ, Filiberti A, Flechtner H, Fleishman SB, de Haes, JC: The European Organization for Research and Treatment of Cancer QLQ-C30: a quality-of-life instrument for use in international clinical trials in oncology. J Natl Cancer Inst 1993, 85:365-76.

9. Osoba D, Aaronson NK, Muller M, Sneeuw K, Hsu MA, Yung WK, Brada M, Newlands E: The development and psychometric validation of a brain cancer quality-of-life questionnaire for use in combination with general cancer-specific questionnaires. Qual Life Res 1996, 5:139-50.

10. Cella DF, Tulsky DS, Gray G, Sarafian B, Linn E, Bonomi A, Silberman M, Yellen SB, Winicour P, Brannon J: The Functional
Assessment of Cancer Therapy scale: development and validation of the general measure. J Clin Oncol 1993, I 1:570-9.

II. Weitzner MA, Meyers CA, Gelke CK, Byrne KS, Cella DF, Levin VA: The Functional Assessment of Cancer Therapy (FACT) scale. Development of a brain subscale and revalidation of the general version (FACT-G) in patients with primary brain tumors. Cancer 1995, 75: ||5|-6|.

12. Cleeland CS, Mendoza TR, Wang XS, Chou C, Harle MT, Morrissey M, Engstrom MC: Assessing symptom distress in cancer patients: the M.D. Anderson Symptom Inventory. Cancer 2000, 89:1634-46.

13. Armstrong TS, Mendoza T, Gning I, Gring I, Coco C, Cohen MZ, Eriksen L, Hsu M, Gilbert MR, Cleeland C: Validation of the M.D. Anderson Symptom Inventory Brain Tumor Module (MDASI-BT). J Neurooncol 2006, 80:27-35.

14. Wellisch DK, Kaleita TA, Freeman D, Cloughesy T, Goldman J: Predicting major depression in brain tumor patients. Psychooncology 2002, II:230-8.

15. Taphoorn, Martin JB, Stupp R, Coens C, Osoba D, Kortmann R, van den Bent, Martin J, Mason W, Mirimanoff RO, Baumert BG, Eisenhauer E, Forsyth P, Bottomley A: Health-related quality of life in patients with glioblastoma: a randomised controlled trial. Lancet Oncol 2005, 6:937-44.

\section{FlOOOPrime}

16. Whitton AC, Rhydderch H, Furlong W, Feeny D, Barr RD: Selfreported comprehensive health status of adult brain tumor patients using the Health Utilities Index. Cancer 1997, 80:258-65.

17. Osoba D, Brada M, Prados MD, Yung WK: Effect of disease burden on health-related quality of life in patients with malignant gliomas. Neuro-oncology 2000, 2:221-8.

18. Salo J, Niemelä A, Joukamaa M, Koivukangas J: Effect of brain tumour laterality on patients' perceived quality of life. J Neurol Neurosurg Psychiatr 2002, 72:373-7.

\section{FIOOOPrime
RECOMMENDED}

19. Gustafsson M, Edvardsson T, Ahlström G: The relationship between function, quality of life and coping in patients with low-grade gliomas. Support Care Cancer 2006, 14:1205-I2.

20. Klein M, Engelberts, Nadine HJ, van der Ploeg, Henk M, KasteleijnNolst Trenité, Dorotheé G A, Aaronson NK, Taphoorn, Martin JB, Baaijen H, Vandertop WP, Muller M, Postma TJ, Heimans JJ: Epilepsy in low-grade gliomas: the impact on cognitive function and quality of life. Ann Neurol 2003, 54:5 I4-20.

\section{FlOOOPrime
RECOMMENDED}

21. Pignatti F, van den Bent, Martin, Curran D, Debruyne C, Sylvester R, Therasse P, Afra D, Cornu P, Bolla M, Vecht C, Karim, Abul BMF: Prognostic factors for survival in adult patients with cerebral low-grade glioma. J Clin Oncol 2002, 20:2076-84.

22. Chang EF, Potts MB, Keles GE, Lamborn KR, Chang SM, Barbaro NM, Berger MS: Seizure characteristics and control following resection in $\mathbf{3 3 2}$ patients with low-grade gliomas. J Neurosurg 2008, 108:227-35.

23. Mainio A, Tuunanen $S$, Hakko $H$, Niemelä $A$, Koivukangas J, Räsänen $P$ : Decreased quality of life and depression as predictors for shorter survival among patients with low-grade gliomas: a follow-up from 1990 to 2003. Eur Arch Psychiatry Clin Neurosci 2006, 256:5|6-2|.

\section{FlOOOPrime} RECOMMENDED

24. Pelletier G, Verhoef MJ, Khatri N, Hagen N: Quality of life in brain tumor patients: the relative contributions of depression, 
fatigue, emotional distress, and existential issues. J Neurooncol 2002, 57:41-9.

\section{FlOOOPrime}

25. Giovagnoli AR, Tamburini M, Boiardi A: Quality of life in brain tumor patients. J Neurooncol 1996, 30:71-80.

26. Klein M, Postma TJ, Taphoorn, MJB, Aaronson NK, Vandertop WP, Muller M, van der Ploeg, HM, Heimans J]: The prognostic value of cognitive functioning in the survival of patients with highgrade glioma. Neurology 2003, 6 I:1796-8.

27. Taphoorn, Martin JB, Klein M: Cognitive deficits in adult patients with brain tumours. Lancet Neurol 2004, 3:I59-68.

28. Meyers CA, Hess KR: Multifaceted end points in brain tumor clinical trials: cognitive deterioration precedes MRI progression. Neuro-oncology 2003, 5:89-95.

\section{FlOOOPrime}

\section{RECOMMENDED}

29. Shaw EG, Rosdhal R, D'Agostino RB, Lovato J, Naughton MJ, Robbins ME, Rapp SR: Phase II study of donepezil in irradiated brain tumor patients: effect on cognitive function, mood, and quality of life. J Clin Oncol 2006, 24:|4|5-20.

30. Meyers CA, Weitzner MA, Valentine AD, Levin VA: Methylphenidate therapy improves cognition, mood, and function of brain tumor patients. J Clin Oncol 1998, 16:2522-7.

31. Boele FW, Douw L, Groot M de, van Thuijl, Hinke F, Cleijne W, Heimans J], Taphoorn, Martin JB, Reijneveld JC, Klein M: The effect of modafinil on fatigue, cognitive functioning, and mood in primary brain tumor patients: a multicenter randomized controlled trial. Neuro-oncology 2013, I5:|420-8.

32. Fox S, Laws ER, Anderson F, Farace E: Complementary therapy use and quality of life in persons with high-grade gliomas. J Neurosci Nurs 2006, 38:212-20.

33. Jalali $R$, Dutta $D$ : Factors influencing quality of life in adult patients with primary brain tumors. Neuro Oncol 2012, I4(Suppl 4):iv8-16.

34. Brown PD, Ballman KV, Rummans TA, Maurer MJ, Sloan JA, Boeve BF, Gupta L, Tang-Wai DF, Arusell RM, Clark MM, Buckner JC: Prospective study of quality of life in adults with newly diagnosed highgrade gliomas. J Neurooncol 2006, 76:283-91.

\section{FlOOOPrime \\ RECOMMENDED}

35. Dutta D, Vanere P, Gupta T, Munshi A, Jalali R: Factors influencing activities of daily living using FIM-FAM scoring system before starting adjuvant treatment in patients with brain tumors: results from a prospective study. J Neurooncol 2009, 94:103-10

36. Mainio A, Hakko H, Niemelä A, Koivukangas J, Räsänen P: Gender difference in relation to depression and quality of life among patients with a primary brain tumor. Eur Psychiatry 2006, 2I:194-9.

\section{FlOOOPrime RECOMMENDED}

37. Budrukkar A, Jalali R, Dutta D, Sarin R, Devlekar R, Parab S, Kakde A: Prospective assessment of quality of life in adult patients with primary brain tumors in routine neurooncology practice. J Neurooncol 2009, 95:413-9.

38. Mainio A, Hakko H, Niemelä A, Tuurinkoski T, Koivukangas J, Räsänen $P$ : The effect of brain tumour laterality on anxiety levels among neurosurgical patients. J Neurol Neurosurg Psychiatr 2003, 74: 1278-82.

\section{FlOOOPrime}

\section{RECOMMENDED}

39. Klein M, Taphoorn MJ, Heimans J], van der Ploeg, HM, Vandertop WP, Smit EF, Leenstra S, Tulleken CA, Boogerd W, Belderbos JS, Cleijne W, Aaronson NK: Neurobehavioral status and health-related quality of life in newly diagnosed high-grade glioma patients. I Clin Oncol 200 I, 19:4037-47.

\section{FlOOOPrime}

RECOMMENDED

40. Crossen JR, Garwood D, Glatstein E, Neuwelt EA: Neurobehavioral sequelae of cranial irradiation in adults: a review of radiationinduced encephalopathy. J Clin Oncol 1994, I 2:627-42.

41. Henriksson R, Bottomley A, Mason W, Saran F, Wick W, Nishikawa R, Saran F, Wick W, Nishikawa R, Cloughesy TF, Carpentier AF, Hoang-Xuan K, Kavan P, Cernea D, Brandes AA, Hilton M, Guijarro AMA, Ravelo A, Chinot OL: Progression-free survival (PFS) and health-related quality of life (HRQoL) in AVAglio, a phase III study of bevacizumab (Bv), temozolomide (T), and radiotherapy (RT) in newly diagnosed glioblastoma (GBM). J Clin Oncol 2013, 3 I (Suppl I5):2005.

42. Litofsky NS, Farace E, Anderson F, Meyers CA, Huang W, Laws ER: Depression in patients with high-grade glioma: results of the Glioma Outcomes Project. Neurosurgery 2004, 54:358-66; discussion 366-7.

43. Sizoo EM, Taphoorn, Martin JB, Uitdehaag B, Heimans JJ, Deliens L, Reijneveld JC, Pasman, $H$ Roeline W: The end-of-life phase of highgrade glioma patients: dying with dignity? Oncologist 2013, I 8: 198-203.

\section{FlOOOPrime}

\section{RECOMMENDED}

44. Walbert T, Khan M: End-of-life symptoms and care in patients with primary malignant brain tumors: a systematic literature review. J Neurooncol 2014, I I 7:2 I7-24

\section{FlOOOPrime
RECOMMENDED}

45. Chochinov HM, Hack T, McClement S, Kristjanson L, Harlos M: Dignity in the terminally ill: a developing empirical model. Soc Sci Med 2002, 54:433-43.

46. Arvold ND, Wang Y, Zigler C, Schrag D, Dominici F: Hospitalization burden and survival among older glioblastoma patients. Neuro-oncology 2014.

\section{FlOOOPrime}

\section{RECOMMENDED}

47. Zhang B, Nilsson ME, Prigerson HG: Factors important to patients' quality of life at the end of life. Arch Intern Med 2012, I 72: | | 33-42.

\section{FlOOOPrime \\ RECOMMENDED}

48. Sizoo EM, Pasman, H Roeline W, Buttolo J, Heimans J], Klein M, Deliens L, Reijneveld JC, Taphoorn, Martin JB: Decision-making in the end-of-life phase of high-grade glioma patients. Eur J Cancer 20I2, 48:226-32

\section{FIOOOPrime
RECOMMENDED}

49. Pace A, Di Lorenzo C, Capon A, Villani V, Benincasa D, Guariglia L, Salvati M, Brogna C, Mantini V, Mastromattei A, Pompili A: Quality of care and rehospitalization rate in the last stage of disease in brain tumor patients assisted at home: a cost effectiveness study. J Palliat Med 2012, I5:225-7.

\section{FIOOOPrime
RECOMMENDED}

50. Pace A, Metro G, Fabi A: Supportive care in neurooncology. Curr Opin Oncol 2010, 22:62I-6.

5I. Sizoo EM, Grisold W, Taphoorn, Martin JB: Neurologic aspects of palliative care: the end of life setting. Handb Clin Neurol 2014, I21:1219-25.

52. Pace A, Villani V, Di Lorenzo C, Guariglia L, Maschio M, Pompili A, Carapella CM: Epilepsy in the end-of-life phase in patients with high-grade gliomas. J Neurooncol 201 3, I I I:83-6.

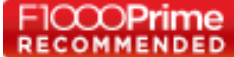


53. Pace $\mathrm{A}, \mathrm{Di}$ Lorenzo $\mathrm{C}$, Guariglia $\mathrm{L}$, Jandolo $\mathrm{B}$, Carapella $\mathrm{CM}$, Pompili A: End of life issues in brain tumor patients. J Neurooncol 2009, $91: 39-43$.

54. Janssens R, van Delden, Johannes JM, Widdershoven, Guy AM: Palliative sedation: not just normal medical practice. Ethical reflections on the Royal Dutch Medical Association's guideline on palliative sedation. J Med Ethics 2012, 38:664-8.

55. Ford E, Catt S, Chalmers A, Fallowfield L: Systematic review of supportive care needs in patients with primary malignant brain tumors. Neuro-oncology 2012, 14:392-404.

\section{FIOOOPrime}

56. Higginson IJ: End-of-life care: lessons from other nations. J Palliat Med 2005, 8(Suppl I):SI6I-73.

57. Wright M, Wood J, Lynch T, Clark D: Mapping levels of palliative care development: a global view. J Pain Symptom Manage 2008, 35:469-85.

FlOOOPrime
58. Lynch T, Connor S, Clark D: Mapping levels of palliative care development: a global update. J Pain Symptom Manage 2013, 45: 1094-106

\section{FlOOOPrime}

RECOMMENDED

59. The Quality of Death: Ranking end-of-life care across the world. [www.eiu.com/sponsor/lienfoundation/qualityofdeath]

60. Jalali R: The palliative care survey results from ASNO. The Asian perspective. Presented at 4th Quadrennial Meeting of the World Federation of Neuro-Oncology: 21 November 2013; California; CA.

6I. Schubart JR, Kinzie MB, Farace E: Caring for the brain tumor patient: family caregiver burden and unmet needs. Neurooncology 2008, 10:61-72.

62. Zelcer S, Cataudella D, Cairney, A Elizabeth L, Bannister SL: Palliative care of children with brain tumors: a parental perspective. Arch Pediatr Adolesc Med 2010, 164:225-30.

63. Kars MC, Grypdonck, Mieke HF, de Korte-Verhoef, Maria C, Kamps WA, Meijer-van den Bergh, Esther M M, Verkerk MA, van Delden, Johannes JM: Parental experience at the end-of-life in children with cancer: 'preservation' and 'letting go' in relation to loss. Support Care Cancer 20I I, 19:27-35. 